\section{Acute Generalized Exanthematous Pustulosis Due to Teicoplanin}

Crespo $\mathrm{J}^{1,3^{*}}$, Lainez-Nuez $\mathrm{A}^{1,3^{*}}$, Cuevas-Bravo $\mathrm{C}^{1,3}$, Tornero $\mathrm{P}^{1,3}$, Mateos-Mayo $\mathrm{A}^{2}$, Rojas-Pérez-Ezquerra $\mathrm{P}^{1,3}$, NogueradoMellado B ${ }^{1,3}$

${ }^{1}$ Allergy Department, University Hospital Gregorio Marañon, Madrid, Spain

${ }^{2}$ Dermatology Department. University Hospital Gregorio Marañon, Madrid, Spain

${ }^{3}$ Gregorio Marañón Health Research Institute, Madrid, Spain

*These authors contributed equally to this work.

J Investig Allergol Clin Immunol 2020; Vol. 30(4): 303-304 doi: 10.18176/jiaci.0501

Key words: Acute generalized exanthematous pustulosis. Patch tests. Psoriasis. Pustules. Teicoplanin.

Palabras clave: Pustulosis exantemática generalizada aguda. Pruebas en parche. Psoriasis. Pústulas. Teicoplanina.

Acute generalized exanthematous pustulosis (AGEP) is a severe, drug-related reaction characterized by acute onset of nonfollicular sterile pustules on erythematous skin, fever $\left(>38^{\circ} \mathrm{C}\right)$, and neutrophilic leukocytosis [1]. It generally appears within 48-72 hours of administration of the drug, although in some cases, symptoms may take up to 3 weeks to appear [2]. The list of drugs that have been associated with AGEP is increasing, although very few cases related to glycopeptides have been reported.

We report the case of a 60 -year-old woman with no personal history of psoriasis or other skin conditions who was admitted to hospital for total knee arthroplasty using a cemented prosthesis (gentamicin and clindamycin). During surgery, she received meropenem, with maintenance for up to 6 days (combined with teicoplanin for the first 4 days, paracetamol, and metamizole). One week after admission, she developed an erythematous rash in the axillary and inguinal folds that was initially treated with fluconazole $50 \mathrm{mg} / 24 \mathrm{~h}$ because of suspected candidal intertrigo. The lesions progressed unfavorably, extending to the thorax, abdomen, and lower limbs, with groups of nonfollicular pustules on an erythematous desquamative base and fever $\left(38^{\circ} \mathrm{C}\right)$. The mucous membranes were not affected. The surgical wound showed no inflammatory signs, and her surgeon ruled out possible postoperative complications. Blood tests showed leukocytosis with neutrophilia (28 000/ $\mu \mathrm{L} ; 91 \%$ neutrophils), elevated acutephase reactants (fibrinogen, $506 \mathrm{mg} / \mathrm{dL}$; C-reactive protein, $6.8 \mathrm{mg} / \mathrm{dL}$ ), and normal renal and hepatic function. Despite treatment with topical and systemic corticosteroids and antihistamines, the patient's condition continued to worsen. Two weeks later, treatment with cyclosporine was started, and the patient improved over the following 2 weeks. A skin biopsy revealed frequent spongiform pustules with no fungal elements in the spinous layer of the epidermis and a perivascular lymphocytic inflammatory infiltrate with moderate eosinophilia in the dermis. These findings were consistent with AGEP. Six months after resolution of AGEP, patch tests were performed using Finn Chambers AQUA patch test strips with clindamycin 5\% (dimethyl sulfoxide [DMSO]), gentamicin $20 \%$ petrolatum (pet), benzylpenicillin $1 \%$ (pet), meropenem 5\% (DMSO), teicoplanin 10\% (DMSO), paracetamol 10\% (pet), metamizole 1\% (pet), and a DMSO control. Readings taken on days 2 and 4 yielded negative results. In addition, intradermal tests performed with benzylpenicilloyl poly-L-lysine as the major determinant at a concentration of $0.04 \mathrm{mg} / \mathrm{mL}$ and a minor determinant mixture (benzylpenicillin sodium, benzylpenicilloic acid, and sodium benzylpenicilloate), amoxicillin, meropenem, vancomycin, teicoplanin, gentamicin, and metamizole yielded negative readings at 24 hours. The result of a single-blind challenge test with meropenem, clindamycin, and paracetamol was negative. Given our high clinical suspicion of AGEP associated with gentamicin or metamizole, a single-blind challenge test with teicoplanin was performed on several consecutive days, with doses increasing up to the therapeutic dose. On the second day of the teicoplanin infusion, after 6 hours of administration (200 mg), the patient developed an erythematous exanthema on the skinfolds, neck, upper limbs, trunk, pinnas, and scalp, with nonfollicular pustules in the occipital region, neck, and back accompanied by fever $\left(39^{\circ}\right)$ (Figure). She was treated with high-dose topical and systemic corticosteroids for 10 days, although despite treatment, no improvement was observed. The reaction eventually affected $90 \%$ of the total body surface area; therefore, the patient was admitted to hospital again for intravenous treatment. Despite the high dosage of systemic corticosteroids, skin involvement persisted for 1-2 weeks, leading us to consider a differential diagnosis with generalized pustular psoriasis (GPP) and initiate weekly treatment with etanercept $50 \mathrm{mg}$. The patient's condition improved slightly. In this case, given the long duration of the clinical course, we decided to perform a skin biopsy, which reflected frequent pustules in the spinous layer of the spongiform-type epidermis. The adjacent horny layer was properly constituted, with no microabscesses or obvious fungal elements, and the thickness of the epidermis was preserved with no other significant

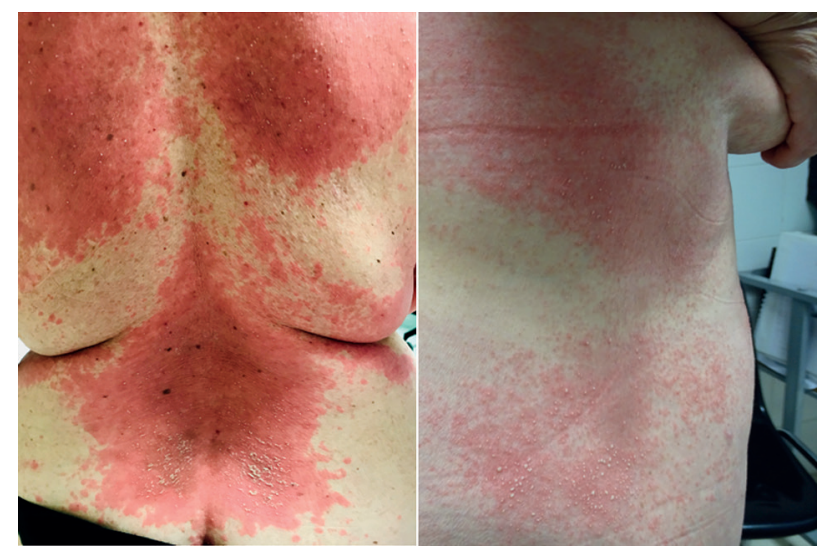

Figure. Erythematous exanthema on skinfolds and trunk with nonfollicular pustules after single-blind challenge test with teicoplanin (200 mg) 
abnormalities. In the underlying dermis, inflammatory perivascular lymphocyte infiltrate was observed with moderate eosinophilia, thus confirming AGEP.

Teicoplanin is a glycopeptide antibiotic that is indicated in infections caused by gram-positive bacteria. It has been increasingly used in recent years and is now a first-line agent during surgery [3]. Immediate allergic reactions to teicoplanin were previously considered extremely rare, although they are more frequent than delayed reactions [4]. In 2001, Chu et al [5] reported the first case of teicoplanin-induced AGEP. Patch testing with teicoplanin was performed 1 month later. Readings made 3 days later were negative. No challenge tests were carried out. In 2011, Turrentine et al [6] reported a case of AGEP induced by telavancin, although no skin tests were performed. In 2015, Mawri et al [2] reported a case of AGEP due to vancomycin with an atypical presentation; however, no allergy work-up was carried out. Sidoroff et al [7] proposed a useful algorithm for the diagnosis of AGEP. In the present case, we recorded an AGEP validation score of 8, thus confirming the diagnosis

In order to diagnose the culprit agent causing the reaction, patch testing with readings on days 2 and 4 is considered useful (sensitivity of up to 58\%) [8]. Nevertheless, intradermal testing with late readings is potentially useful. Both tests yielded negative results.

Typically self-limiting in nature, AGEP tends to resolve spontaneously with cessation of the offending drug. In this case, the differential diagnosis was with GPP, which is a rare variant of psoriasis of unknown etiology that usually causes generalized erythematous and pustular rash associated with occasional severe systemic reactions. It can develop in patients with or without a previous personal history of psoriasis and can be triggered by factors such as infection or drugs. The rash of GPP is morphologically identical to that of AGEP, although the distribution pattern is more widespread and the rash and fever persist longer $[9,10]$. AGEP and GPP have been considered disorders on the same spectrum and can overlap. In this case, GPP was ruled out by skin biopsy.

Teicoplanin is a recognized but very rare cause of AGEP. The case we report is unique, not only because teicoplanin was confirmed to be the culprit medication with an oral challenge test, but also because of the atypically non-self-limiting progress of the cutaneous eruption that required elevated doses of systemic corticosteroids, cyclosporine, and etanercept. In all cases, it is important to take into account other entities that could mimic AGEP. It is also important to consider the potentially long course and severity of AGEP.

\section{Funding}

This study was funded by the National Health System.

\section{Conflicts of Interest}

The authors declare that they have no conflicts of interest.

\section{References}

1. Speeckaert MM, Speeckaert R, Lambert J, Brochez L. Acute generalized exanthematous pustulosis: an overview of the clinical, immunological and diagnostic concepts. Eur J Dermatol. 2010;20:425.

2. Mawri S, Jain T, Shah J, Hurst G, Swiderek J. Vancomycininduced acute generalized exanthematous pustulosis (AGEP) masquerading septic shock-an unusual presentation of a rare disease. J Intensive Care. 2015;10;3:47

3. Savic LC, Garcez T, Hopkins PM, Harper NJN, Savic S. Teicoplanin allergy - an emerging problem in the anaesthetic allergy clinic. Br J Anaesth. 2015;115:595-600.

4. Butler MS, Hansford KA, Blaskovich MAT, Halai R, Cooper MA. Glycopeptide antibiotics: Back to the future. J Antibiot (Tokyo). 2014;67:631-44.

5. Chu $C Y$, Wu J, Jean SS, Sun CC. Acute Generalized Exanthematous Pustulosis due to Teicoplanin. Dermatology. 2001;202:141-2.

6. Turrentine JE, Dharamsi JW, Miedler JD, Pandya AG. Acute generalized exanthematous pustulosis (AGEP) caused by telavancin. J Am Acad Dermatol. 2011;65:e100-e101

7. Sidoroff A, Halevy S, Bavinck JN, Vaillant L, Roujeau JC. Acute generalized exanthematous pustulosis (AGEP) a clinical reaction pattern. J Cutan Pathol. 2001;28:113.

8. Phillips EJ, Bigliardi P, Bircher AJ, Broyles A, Chang YS, Chung $\mathrm{WH}$, et al. Controversies in drug allergy: Testing for delayed reactions. J Allergy Clin Immunol. 2019;143:66-73.

9. Martin-Hernandez E, García-Alvarez S. Pustulosis aguda generalizada: reporte de caso. Med Cutan Iber Lat Am. 2018;46:38-41.

10. Unal S, Ikizoglu G, Demirkan F, Kaya TI. Teicoplanin-induced skin eruption. Int J Dermatol. 2002;41:948-9.

- Manuscript received September 30, 2019; accepted for publication February 19, 2020.

Ana Láinez Nuez

Allergy Department Hospital General Universitario Gregorio Marañón

Dr. Esquerdo, 46 28007 Madrid, Spain E-mail: alainuez@gmail.com 